\title{
Growth and differentiation factor 15 is secreted by skeletal muscle during exercise and promotes lipolysis in humans
}

\author{
Claire Laurens, ${ }^{1,2,3}$ Anisha Parmar, ${ }^{1,2}$ Enda Murphy, ${ }^{4}$ Deborah Carper, ${ }^{1,2}$ Benjamin Lair, ${ }^{1,2}$ \\ Pauline Maes, ${ }^{3}$ Julie Vion, ${ }^{1,2}$ Nathalie Boulet, ${ }^{1,2}$ Coralie Fontaine, ${ }^{1,2}$ Marie Marquès, ${ }^{1,2}$ \\ Dominique Larrouy, ${ }^{1,2}$ Isabelle Harant,, ${ }^{1,2}$ Claire Thalamas, , ${ }^{1,5,6}$ Emilie Montastier, ${ }^{1,2,6}$ \\ Sylvie Caspar-Bauguil,, ${ }^{1,2,6}$ Virginie Bourlier, ${ }^{1,2}$ Geneviève Tavernier, ${ }^{1,2}$ Jean-Louis Grolleau, ${ }^{6}$ \\ Anne Bouloumié, ${ }^{1,2}$ Dominique Langin,, ${ }^{1,2,6}$ Nathalie Viguerie, ${ }^{1,2}$ Fabrice Bertile, ${ }^{3}$ Stéphane Blanc, ${ }^{3}$ \\ Isabelle de Glisezinski, ${ }^{1,2,6}$ Donal $0^{\prime}$ Gorman, ${ }^{4}$ and Cedric Moro ${ }^{1}$ \\ IInserm, UMR1048, Institute of Metabolic and Cardiovascular Diseases, Toulouse, France. Institute of Metabolic and \\ Cardiovascular Diseases, University of Toulouse, Paul Sabatier University, UMR1048, Toulouse, France. ${ }^{3}$ CNRS UMR7178, \\ Institut Pluridisciplinaire Hubert Curien, Strasbourg University, Strasbourg, France. ${ }^{4}$ School of Health and Human \\ Performance, Dublin City University, Dublin, Ireland. ${ }^{5}$ Inserm, Clinical Investigation Center CIC 1436, Toulouse, France. \\ ${ }^{6}$ Departments of Biochemistry and Nutrition, Physiology, Plastic Surgery and Clinical Investigation Center CIC 1436, \\ Toulouse University Hospitals, Toulouse, France.
}

\begin{abstract}
We hypothesized that skeletal muscle contraction produces a cellular stress signal, triggering adipose tissue lipolysis to sustain fuel availability during exercise. The present study aimed at identifying exercise-regulated myokines, also known as exerkines, able to promote lipolysis. Human primary myotubes from lean healthy volunteers were submitted to electrical pulse stimulation (EPS) to mimic either acute intense or chronic moderate exercise. Conditioned media (CM) experiments with human adipocytes were performed. CM and human plasma samples were analyzed using unbiased proteomic screening and/or ELISA. Real-time qPCR was performed in cultured myotubes and muscle biopsy samples. CM from both acute intense and chronic moderate exercise increased basal lipolysis in human adipocytes. Growth and differentiation factor 15 (GDF15) gene expression and secretion increased rapidly upon skeletal muscle contraction. GDF15 protein was upregulated in CM from both acute and chronic exercise-stimulated myotubes. We further showed that physiological concentrations of recombinant GDF15 protein increased lipolysis in human adipose tissue, while blocking CDF15 with a neutralizing antibody abrogated EPS CM-mediated lipolysis. We herein provide the first evidence to our knowledge that GDF15 is a potentially novel exerkine produced by skeletal muscle contraction and able to target human adipose tissue to promote lipolysis.
\end{abstract}

Conflict of interest: The authors have declared that no conflict of interest exists.

Copyright: () 2020, American Society for Clinical Investigation.

Submitted: July 17, 2019

Accepted: February 20, 2020

Published: March 26, 2020.

Reference information: /CI Insight. 2020;5(6):e131870.

https://doi.org/10.1172/jci.

insight.131870.

\section{Introduction}

Exercise improves metabolic health and prevents the complications of obesity and type 2 diabetes $(1,2)$. This is partly due to the release of secreted factors by skeletal muscle, i.e., myokines, which can virtually target all organs remotely (3). Over the last decade, hundreds of proteins secreted by skeletal muscle have been identified, as reviewed in detail elsewhere (4-7). However, very few myokines have been shown to target white adipose tissue, particularly in humans. In a previous clinical study, we observed a remarkably enhanced in situ and systemic lipolytic response in lean healthy endurance-trained subjects performing a high-intensity exercise the day after an exhaustive glycogen-depleting exercise compared with participants that rested (8). This greater lipolytic response could not be explained by significant changes in classically known lipolytic stimuli, such as increased catecholamines, atrial natriuretic peptide, growth hormone, cortisol, and IL-6 or a decrease in circulating insulin during exercise. We therefore hypothesized that skeletal muscle contraction in the context of acute high-intensity exercise and chronic moderate exercise may produce a cellular stress signal capable of increasing adipose tissue lipolysis to sustain fuel availability and delay muscle glycogen depletion. 
Recently, growth and differentiation factor 15 (GDF15) emerged as a biomarker of cellular stress that can be produced by a number of organs, such as lung, kidney, and liver (9-11). GDF15, also known as macrophage inhibitory cytokine-1 (MIC-1), is a stress-induced cytokine and an ancient member of the TGF- $\beta$ superfamily. High levels of circulating GDF15 have been found in various diseases, such as cancer, heart failure, and mitochondrial diseases $(12,13)$. Very recent studies demonstrate a major role of GDF15 in appetite suppression through noncanonical brain neuronal networks in the brainstem, such as the area postrema and the nucleus tractus solitaries (14-17). Injection of recombinant GDF15 protein in mice fed a high-fat diet (HFD) induces robust weight loss (16). Similarly GDF15-overexpressing mice are resistant to HFD-induced obesity and metabolic disturbances (18). These effects appear largely mediated by food intake suppression through the GDF15 cognate receptor GFRAL (GDNF receptor $\alpha$-like), which heterodimerizes with a coreceptor called RET $(15,17)$. RET encodes a tyrosine kinase receptor for members of the glial cell line-derived neurotrophic factor (GDNF) family (15). In mice, GFRAL expression appears strictly confined to the area postrema and the nucleus of the tractus solitary. It is suggested that GDF15 behaves as a metabolic stress signal in response to nutritional stress to trigger conditioned taste aversion (11).

We herein describe GDF15 as a potentially novel exerkine for peripheral crosstalk between skeletal muscle and adipose tissue. We show that conditioned media (CM) from exercised human myotubes activate lipolysis in human adipocytes in vitro. Through proteomic screening of the CM, we next identified GDF15 as an exercise-regulated myokine that is rapidly released upon muscle contraction. Neutralization of GDF15 in CM abolishes lipolysis, while treatment of adipose tissue explants with recombinant GDF15 promotes lipolysis. Collectively, we herein provide what we believe to be the first evidence that GDF15 is produced by skeletal muscle upon contraction and that it is able to promote lipolysis in human adipose tissue.

\section{Results}

CM from exercised myotubes trigger lipolysis in adipocytes. The physiological and metabolic adaptations of skeletal muscle to exercise are well known (1). Using electrical pulse stimulation (EPS) of human myotubes to induce forced contraction, we modeled two exercise paradigms: an acute high-intensity exercise model (EPS3h) and a chronic moderate exercise training model (EPS24h). EPS activates canonical exercise signaling pathways, such as p38 MAPK and $\mathrm{Ca}^{2+} /$ calmodulin-dependent protein kinase II (CaMKII) for up to 48 hours (Supplemental Figure 1A; supplemental material available online with this article; https://doi.org/10.1172/jci. insight.131870DS1). As expected, EPS3h, which mimics an acute high-intensity exercise, induced a pronounced glycogen depletion (Figure $1 \mathrm{~A})$ and concomitant increase in lactate production $(\sim 4$-fold, $P<0.01)$ (Figure 1B). Consistent with this type of exercise, no major change in glucose oxidation rate was observed (Figure 1C), while the lipid oxidation rate was reduced by 35\% (Figure 1D). EPS3h also induced gene expression of classically known myokines, such as IL-6, IL-15, and FGF21 (Supplemental Figure 1B).

We further found that EPS24h, which mimics chronic moderate exercise training, did not significantly reduced glycogen content (Figure $1 \mathrm{E})$ while increasing basal glycogen storage capacity $(\sim 1.5$-fold, $P<0.05)$ (Figure $1 \mathrm{~F})$, glucose oxidation rate $(\sim 2$-fold, $P<0.05)$ (Figure $1 \mathrm{G})$, and lipid oxidation rate $(\sim 3$-fold, $P<$ $0.05)$ (Figure 1H). EPS24h also increased lactate production (2.2-fold, $P<0.01$ ), but to a lesser degree than EPS3h (Supplemental Figure 2A). EPS24h increased the IL-6 mRNA level ( $\sim 3$-fold, $P<0.01$ ), slightly induced myostatin, and decreased fibronectin type III domain-containing 5 (FNDC5) and brain-derived neurotrophic factor (BDNF) mRNA levels (Supplemental Figure 1C). To test our hypothesis that skeletal muscle contraction produces a cellular stress signal that triggers lipolysis to sustain fuel availability during exercise, we next incubated human multipotent adipose-derived stem (hMADS) adipocytes with CM of EPS-stimulated myotubes (Figure 1I). Interestingly, CM from EPS3h-stimulated myotubes induced a modest increase of basal lipolysis, as reflected by glycerol production $(\sim 1.3$-fold, $P<0.001)$ in the absence of any lipolytic stimuli (Figure $1 \mathrm{~J}$ ). Similarly, CM from EPS24h-stimulated myotubes induced a more robust basal lipolysis ( 3-fold, $P<0$.001) (Figure 1K). Similarly, CM from EPS24h induced a significant increase of NEFA release (1.7-fold, $P<0.05)$ (Supplemental Figure $2 \mathrm{~B})$.

In summary, exercise-mediated skeletal muscle contraction produces secreted factors able to activate lipolysis in adipocytes in vitro.

GDF15 is a potentially novel exerkine rapidly induced by skeletal muscle contraction. To identify potential secreted proteins produced by skeletal muscle contraction, we performed a proteomic screen of CM from EPS-stimulated and nonstimulated myotubes (Figure 2, A and D). We quantified 1311 classically 
A

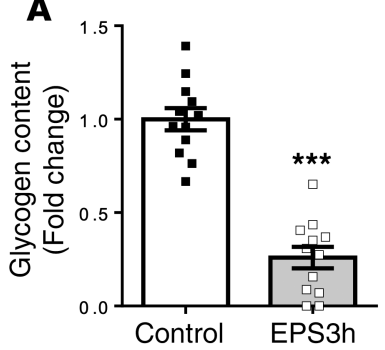

E

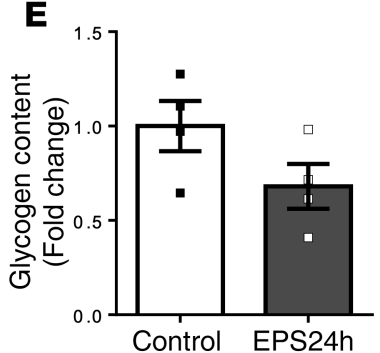

I

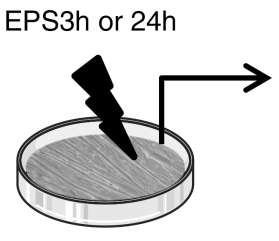

Myotubes
B
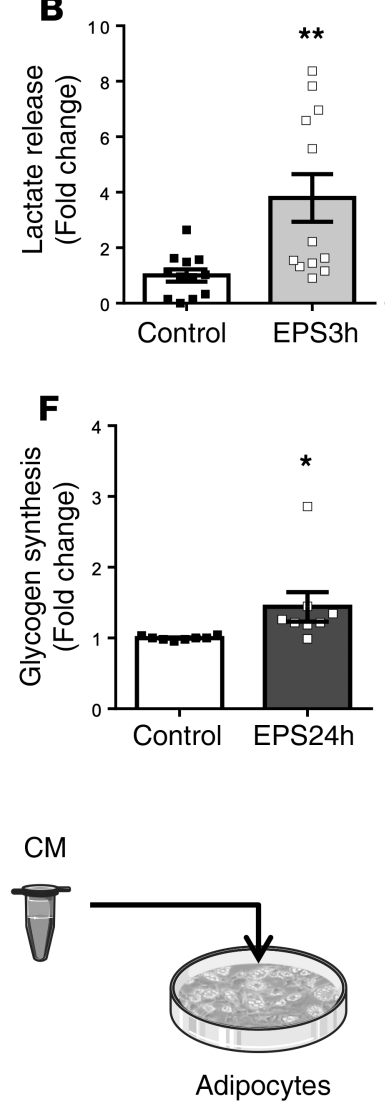
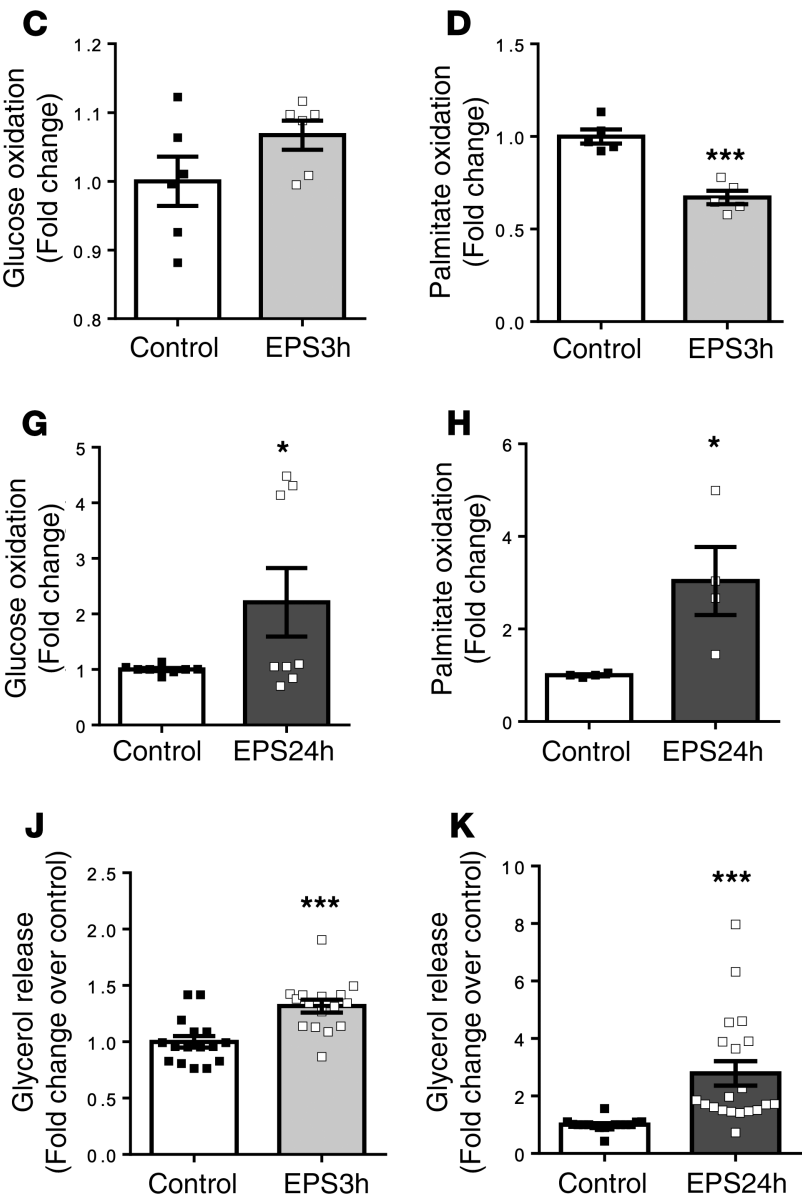

Figure 1. Acute intense and chronic moderate exercise model validation in human myotubes. (A-D) Acute intense exercise model, EPS3h: (A) total glycogen content, (B) lactate secretion, (C) glucose oxidation rate, and (D) palmitate oxidation rate in human myotubes cultured for 5 days submitted to 3 hours of EPS to mimic an acute high-intensity exercise in vitro. Data are expressed as mean $\pm \operatorname{SEM}(n=8)$. ${ }^{*} P<0.05,{ }^{* *} P<0.01,{ }^{* * *} P<0.001$ compared with control by 2 -tailed unpaired Student's $t$ test. (E-H) Chronic moderate exercise model, EPS24h: (E) total glycogen content, (F) basal glycogen synthesis rate, (G) glucose oxidation rate, and $(\mathbf{H})$ palmitate oxidation rate in human myotubes cultured for 5 days submitted to 24-hour EPS to mimic moderate chronic exercise training in vitro. Data are expressed as mean \pm SEM $(n=8) .{ }^{*} P<0.05$ compared with control by 2 -tailed unpaired Student's $t$ test. (I-K) Conditioned media experiments between human myotubes and human adipocytes: (I) experimental design, (J) conditioned media of EPS3h-stimulated myotubes, and (K) conditioned media of EPS24hstimulated myotubes were applied on differentiated hMADS adipocytes for 24 hours to measure glycerol output, a surrogate of lipolysis. Data are expressed as mean \pm SEM $(n=16-20) .{ }^{* * *} P<0.01$ compared with control by 2 -tailed unpaired Student's $t$ test.

and nonclassically secreted proteins. Among the significantly upregulated proteins, GDF15 caught our attention (Figure 2A), as recent studies described GDF15 as a novel metabolic hormone (14-17). GDF15 protein levels were increased by about 2.5 -fold in EPS3h CM (Figure 2B). We confirmed this finding by ELISA: GDF15 increased from $21.7 \pm 2.1 \mathrm{pg} / \mathrm{ml}$ to $29.6 \pm 3.4 \mathrm{pg} / \mathrm{ml}$ in EPS3h CM $(P<$ 0.01 ) (Figure 2C). Importantly, we also identified GDF15 as a significantly upregulated protein in EPS24h CM (3-fold, $P<0.05$ ) (Figure 2, D-F). The concentration of GDF15 in EPS24h CM ranged from 19.4 to $110.9 \mathrm{pg} / \mathrm{ml}$ and was nearly 2 -fold higher $(41.2 \pm 4.0$ vs. $21.7 \pm 2.1 \mathrm{pg} / \mathrm{ml})$ than that in EPS3h CM. The time course of EPS in human primary myotubes showed that GDF15 was significantly upregulated within 1 hour (Figure 2G). Overall, we here identify GDF15 as a potentially novel exercise-regulated myokine.

GDF15 gene expression is responsive to skeletal muscle contraction and exercise mimetics. Since GDF15 is a stress-responsive hormone, we tested whether acute EPS can modulate GDF15 mRNA levels. We observed a rapid upregulation of GDF15 mRNA levels within 30 minutes of exposure to EPS (Figure 3A). A second peak of mRNA levels was observed at 3 hours of EPS. In agreement with protein secretion data, we observed a significant upregulation of GDF15 mRNA levels for EPS3h-stimulated myotubes (1.7-fold, $P<$ 0.01) (Figure 3B). We similarly observed an upregulation of GDF15 mRNA levels in EPS24h-stimulated myotubes (Figure $3 \mathrm{C}$ ). Since exercise activates a complex integrative response, including neuroendocrine 
A

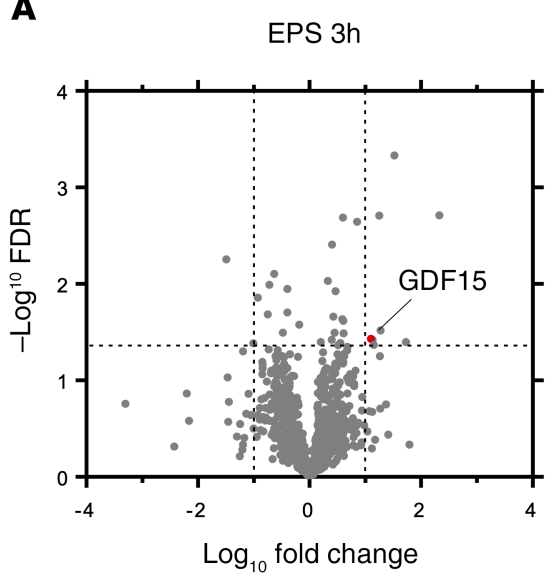

D

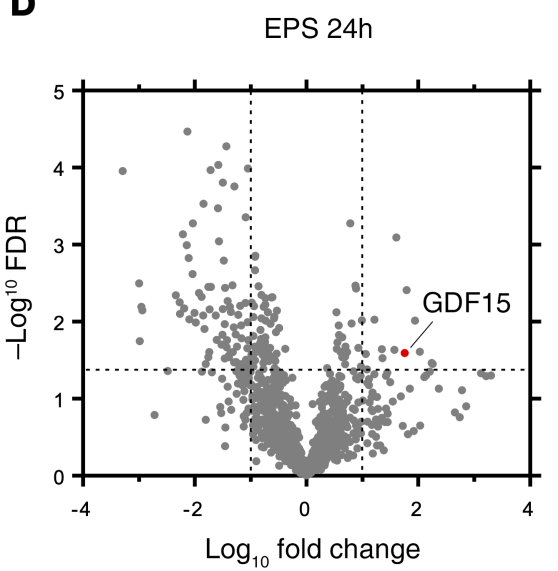

B

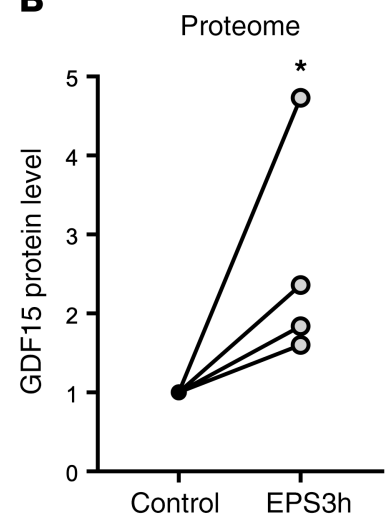

E

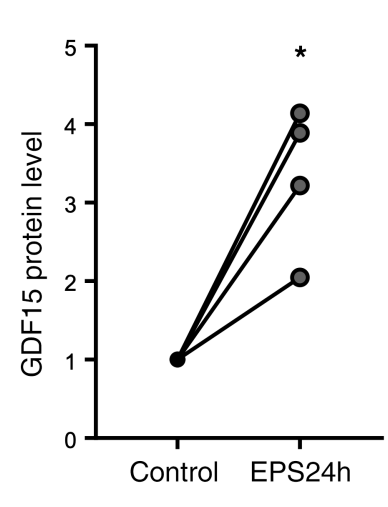

C

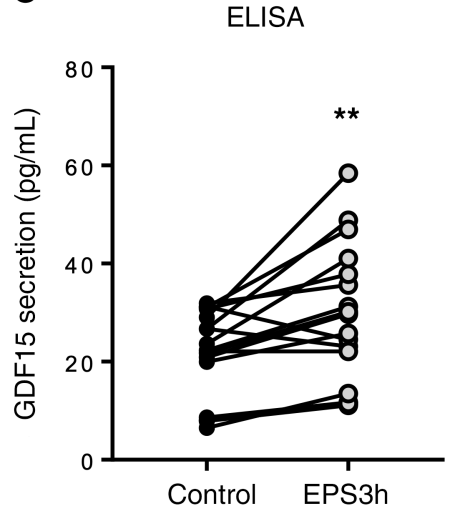

$\mathbf{F}$

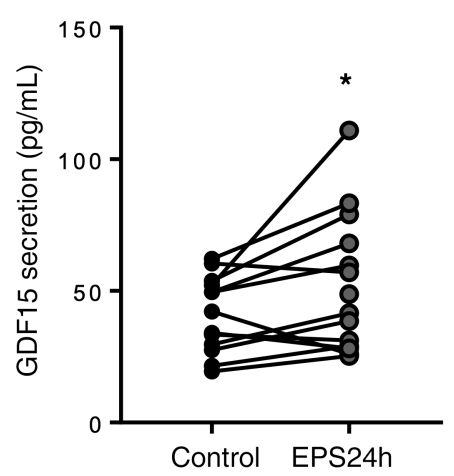

G

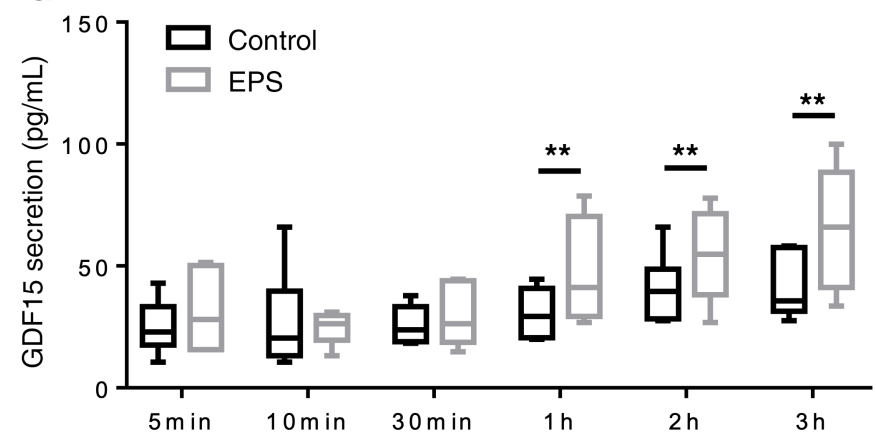

Figure 2. Identification of GDF15 as an exercise-regulated myokine. (A-C) Proteomic screen of conditioned media from EPS3h-stimulated myotubes: (A) Volcano plot of the unbiased proteomic screen reveals GDF15 as a significantly upregulated protein in conditioned media, (B) quantification of GDF15 protein in media from the protein screen, and (C) quantification of GDF15 protein secretion by ELISA in human myotubes cultured for 5 days submitted to 3 hours of EPS to mimic an acute intense exercise in vitro. Data are expressed as mean \pm SEM $(n=16) .{ }^{*} P<0.05,{ }^{* *} P<0.01$ compared with control by 2-tailed paired Student's $t$ test. (D-F) Proteomic screen of conditioned media from EPS24h-stimulated myotubes: (D) Volcano plot of the unbiased proteomic screen reveals GDF15 as a significantly upregulated protein in conditioned media, (E) quantification of GDF15 protein in media from the protein screen, and (F) quantification of GDF15 protein secretion by ELISA in human myotubes cultured for 5 days submitted to 24-hour EPS to mimic a moderate chronic exercise training in vitro. Data are expressed as mean $\pm \operatorname{SEM}(n=16) .{ }^{*} P<0.05$ compared with control by 2-tailed paired Student's $t$ test. (G) Time course of CDF15 protein secretion in acutely EPS-stimulated myotubes. Data are expressed as mean $\pm \operatorname{SEM}(n=6)$. ${ }^{* *} P<0.01$ compared with control by 2-way ANOVA followed by a Bonferroni post hoc test.

changes beyond muscle contraction, we assessed the influence of exercise mimetics on GDF15 mRNA levels. Forskolin, which increases intracellular levels of the second messenger cAMP, did not change GDF15 gene expression (Figure 3D), while, interestingly, both ionomycin, which facilitates calcium release (Figure $3 \mathrm{E}$ ), and the peroxisome proliferator-activated receptor $\beta$ agonist GW0742 (Figure 3F) increased GDF15 mRNA levels $(1.5$-2-fold, $P<0.01)$. We next investigated GDF15 mRNA levels in muscle biopsy samples 
A
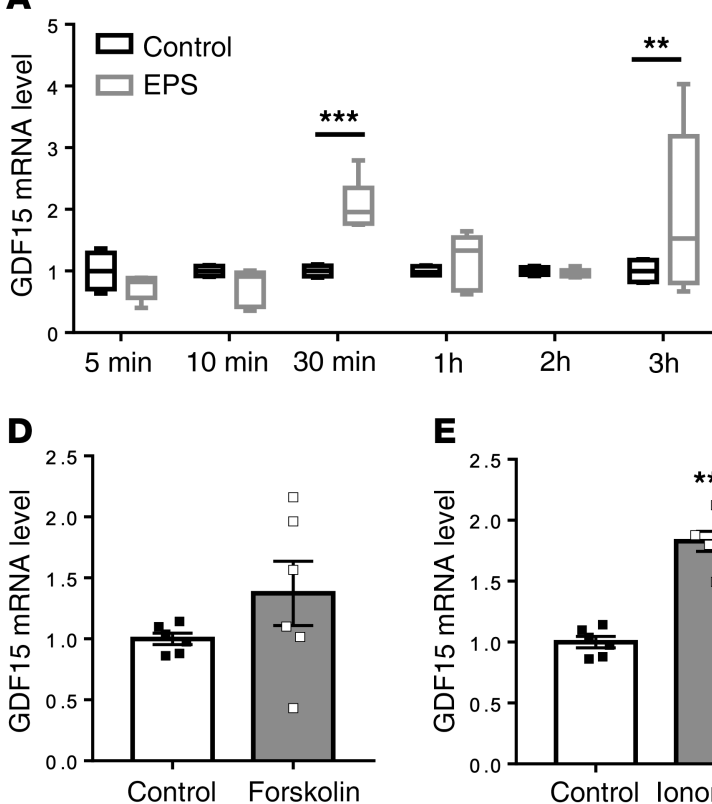

E

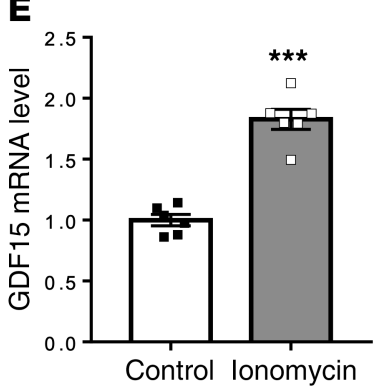

B

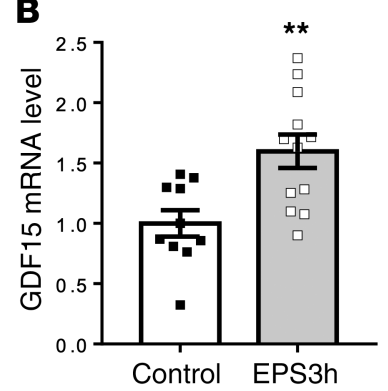

F

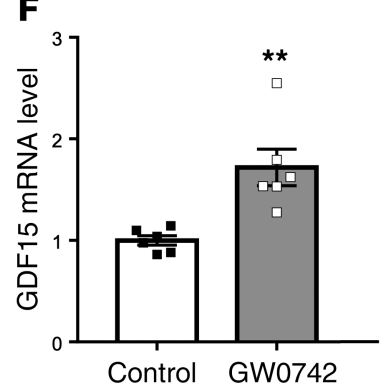

C
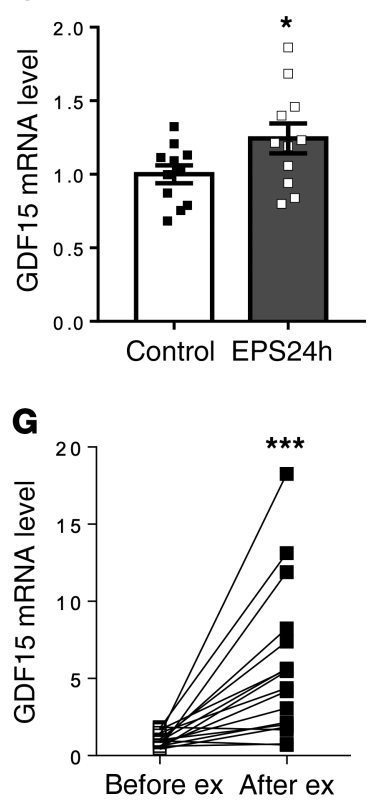

Figure 3. GDF15 gene expression changes in contracting skeletal muscle. (A) Time course of GDF15 mRNA level in acutely EPS-stimulated myotubes. Data are expressed as mean \pm SEM $(n=6)$. ${ }^{* *} P<0.01,{ }^{* *} P<0.001$ compared with control by 2-way ANOVA followed by a Bonferroni post hoc test. (B) GDF15 mRNA level in EPS3h-stimulated myotubes and (C) in EPS24h-stimulated myotubes. Data are expressed as mean \pm SEM $(n=12)$. ${ }^{*} P<0.05$, ** $P<0.01$ compared with control by 2-tailed unpaired Student's $t$ test. (D-F) GDF15 mRNA level changes in response to exercise mimetics: human myotubes cultured for 5 days were treated for 3 hours with (D) $10 \mu \mathrm{M}$ forskolin, (E) $4 \mu \mathrm{M}$ ionomycin, (F) and the PPAR $\delta$ agonist GW0742 (100 nM). Data are expressed as mean \pm SEM $(n=6) .{ }^{* *} P<0.01,{ }^{* *} P<0.001$ compared with control by 2-tailed unpaired Student's $t$ test. (C) GDF15 mRNA level changes in the muscle vastus lateralis biopsy samples taken before and immediately after a 1-hour acute exercise bout in lean healthy volunteers (human study 1). Data are expressed as mean \pm SEM $(n=18) .{ }^{* *} P<0.001$ compared with control by 2 -tailed paired Student's $t$ test.

from lean healthy individuals in response to 1 hour of acute exercise at $55 \%$ peak power output on a bicycle ergometer (human study 1). We found that GDF15 mRNA levels increased up to 18-fold in skeletal muscle in response to exercise (Figure 3G).

GDF15 plasma levels in response to exercise, obesity, and lifestyle. Since our in vitro work revealed GDF15 as a exerkine, we measured GDF15 in plasma of lean healthy individuals randomly assigned in a crossover design to either 1 hour of endurance exercise performed at $60 \%$ of $\mathrm{VO}_{2}$ peak or sprint interval exercise consisting of 7 repetitions of 30 seconds at $130 \%$ of maximal aerobic power (human study 2) (Figure 4, A-C). In line with a recent report (19), we observed that both types of exercise, endurance (Figure 4A) and sprint interval (Figure $4 \mathrm{~B})$, augmented plasma GDF15 circulating levels. Interestingly, the exercise response (exercise minus rest) tended to be lower $(P=0.07)$ for the sprint interval exercise, which produced overall a lower energy expenditure and energetic stress, despite a greater power output during very short periods of time ( $30 \mathrm{~s})$ (Figure 4C).

We further show that acute endurance exercise ( $45 \mathrm{~min}$ at $50 \% \mathrm{VO}_{2 \max }$ ) raised plasma GDF15 levels in both middle-aged and elderly obese subjects (human study 3) (Figure 4, D and E). Plasma GDF15 levels were measured at rest and immediately after exercise at baseline and in response to an 8-week lifestyle intervention program combining moderate calorie restriction and aerobic and resistance exercise. Obese subjects lost in average $5.1 \pm 0.5 \mathrm{~kg}$ after lifestyle intervention (data not shown). An exercise-induced increase of plasma GDF15 was observed in both groups at baseline and after lifestyle intervention (Figure $4, \mathrm{D}$ and $\mathrm{E}$ ). When combining all subjects, we observed a slight but significant increase of plasma GDF15 levels in response to lifestyle intervention-induced weight loss (Figure 4F). This increase of plasma GDF15 tended to be positively correlated with lifestyle intervention-induced weight loss in elderly obese subjects $(\mathrm{r}=0.66 ; P=0.07)$. Lean healthy and middle-aged obese subjects had plasma GDF15 levels within the same range (Figure 4G), while BMI-matched elderly obese subjects had higher circulating levels of GDF15 compared with middle-aged obese individuals (Figure 4H). Consistent with this finding, we observed a significant positive correlation between resting plasma GDF15 levels and age in human study $3(r=0.512, P=0.043)$. Interestingly, no sex difference in resting plasma GDF15 levels was found in human study 4 (men, $475 \pm 56 \mathrm{pg} / \mathrm{ml}$ vs. women, $472 \pm 46 \mathrm{pg} / \mathrm{ml}$ ). 
A

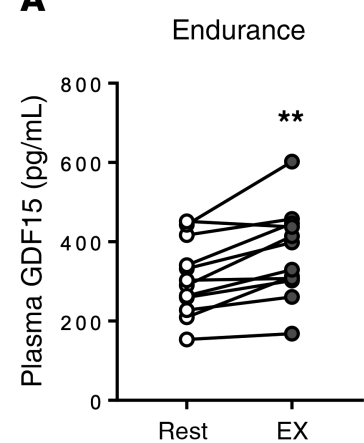

D

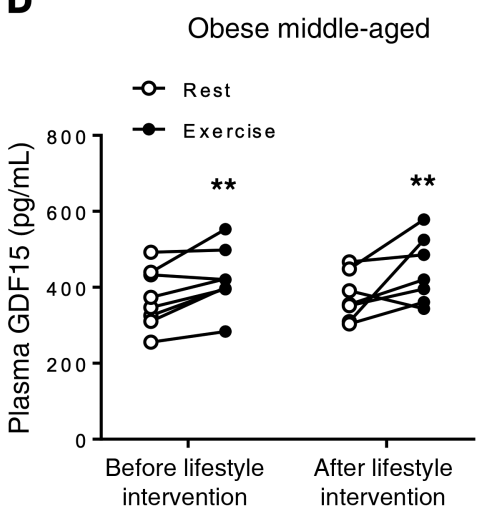

$\mathbf{F}$

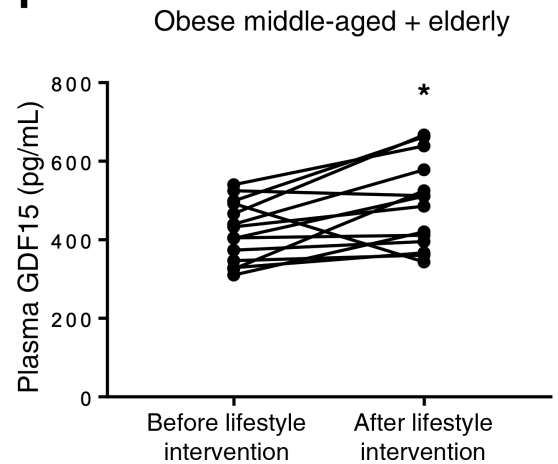

B

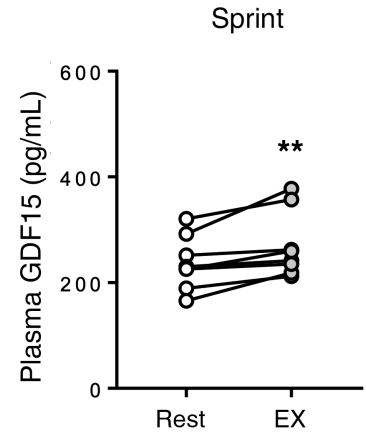

C

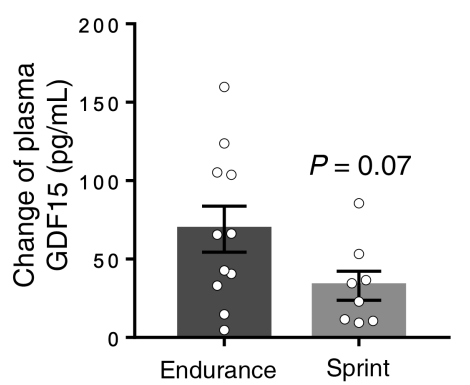

E

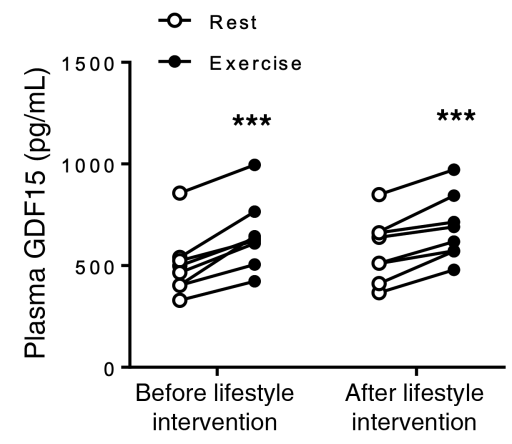

G

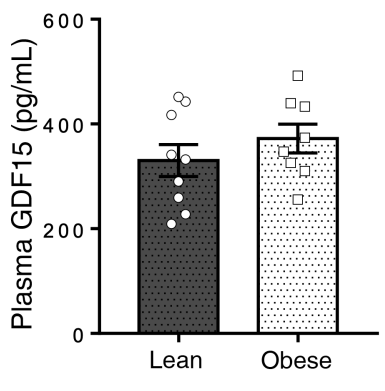

H

Figure 4. Changes in plasma GDF15 levels with exercise, obesity and lifestyle intervention. (A-C) Human study 2: plasma GDF15 changes in lean healthy volunteers in response to (A) endurance exercise for 1 hour at $60 \% \mathrm{VO}_{2 \mathrm{max}}$, (B) sprint interval exercise of 7 repetitions at $130 \%$ of maximal workload, and (C) delta change from rest to exercise during endurance versus sprint interval exercise. Data are expressed as mean \pm SEM ( $n=8-15$ ). ${ }^{* *} P<0.01$ compared with control by 2-tailed paired Student's $t$ test. (D-F) Human study 3: plasma GDF15 changes in obese middle-aged (D) and elderly (E) subjects at baseline and in response to a 8 -week lifestyle intervention-induced weight loss program. Data are expressed as mean \pm SEM ( $n=8$ ). ${ }^{* *} P<0.01,{ }^{* * *} P<$ 0.001 compared with control by 2-tailed paired Student's $t$ test. (F) Plasma GDF15 changes in response to lifestyle intervention-induced weight loss in obese individuals (all subjects). Data are expressed as mean \pm SEM $(n=16)$. ${ }^{*} P<0.05$ compared with control by 2 -tailed paired Student's $t$ test. (G) Plasma GDF15 levels in lean healthy (study 2) versus middle-aged obese individuals (study 3 ). (H) Plasma GDF15 levels in middle-aged obese versus elderly obese individuals (study 3). ${ }^{*} P<0.05$ compared with Young by 2-tailed unpaired Student's $t$ test.

Collectively, we here show that plasma GDF15 responds to various modalities of exercise in lean and obese individuals and increases with age.

GDF15 targets human adipose tissue to promote lipolysis. We noted a positive correlation between plasma glycerol and GDF15 level in all obese subjects investigated at rest and during exercise (human study 3) (Supplemental Figure 3A). We further observed a nearly significant positive relationship ( $\mathrm{r}=0.35, P=$ 0.067 ) between changes in plasma GDF15 levels and changes in BMI in obese individuals in response to very low calorie diet-induced weight loss (human study 4) (Supplemental Figure 3B). We therefore hypothesized that GDF15 was able to target white adipose tissue to induce lipolysis and weight loss. We first examined mRNA levels of GDF15 receptors GFRAL and RET in human abdominal adipose 
Table 1. Relationship between biological and anthropometric variables and subcutaneous abdominal adipose tissue GFRAL and RET mRNA levels

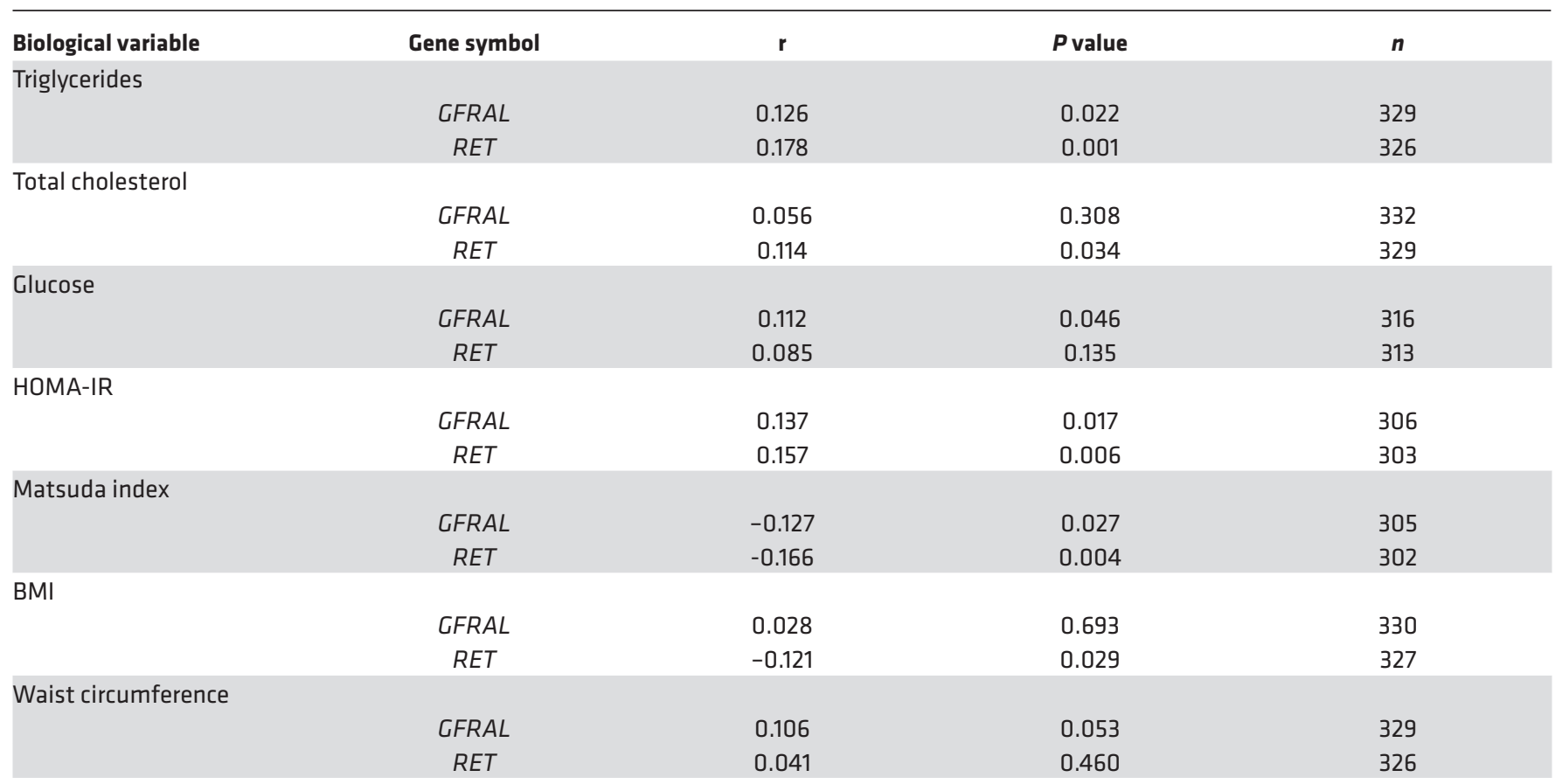

These data were obtained from human study 4. HOMA-IR, homeostatic model assessment of insulin resistance; r, Pearson correlation coefficients. The Matsuda index was calculated as 10,000/square root of [(fasting glucose $\times$ fasting insulin) $\times$ (mean glucose $\times$ mean insulin during OGTT)]

tissue biopsy samples (human study 4). We found substantial expression of the GDF15 cognate receptor GFRAL in the whole adipose tissue fraction as well as in isolated adipocytes (Supplemental Figure 4A). Interestingly, GFRAL expression was virtually null in whole human muscle tissue. The coreceptor RET followed a similar pattern of expression as GFRAL in adipose tissue, except for whole skeletal muscle tissue, where its expression was comparable to whole adipose tissue (Supplemental Figure 4B). As both GFRAL and RET were expressed to comparable levels in adipocytes and adipose stroma vascular cells, we further examined expression of both GDF15 receptors in sorted stromal cells. We observed that both GFRAL (Supplemental Figure 4C) and RET (Supplemental Figure 4D) were predominantly expressed in preadipocytes, again confirming a metabolic role of GDF15 in adipocytes. Neither GFRAL (Supplemental Figure 4E) nor RET (Supplemental Figure 4F) adipose gene expression was influenced by obesity grade. However, they were both associated with a number of biological and clinical variables, such as plasma triglycerides, HOMA-IR, and Matsuda index, while adipose RET mRNA levels were inversely related to BMI (Table 1).

We then investigated the effect of recombinant human GDF15 (rhGDF15) protein on lipolysis in human abdominal adipose tissue explants. rhGDF15 significantly modestly increased lipolysis at the physiological concentration of $1 \mathrm{ng} / \mathrm{ml}(\sim 10 \%, P<0.05)$, reflected by both glycerol (Figure $5 \mathrm{~A})$ and NEFA release (Figure $5 \mathrm{~B}$ ). This lipolytic response was weak compared with those observed for forskolin and the nonselective $\beta$-adrenergic agonist isoprenaline, which increased lipolysis to about 2 - to 3 -fold. A higher supraphysiological concentration of rhGDF15 at $100 \mathrm{ng} / \mathrm{ml}$ did not further raised basal lipolysis $(\sim 11 \%, P<0.05)$ in adipose explants from 6 independent donors (Figure 5, C and D). Even more remarkable, we demonstrate that blocking GDF15 with a neutralizing IgG antibody of hGDF15 completely abrogates the lipolytic response of EPS24h CM (Figure 5E), thus indicating that GDF15 likely triggers most, if not all, the EPS24h CM lipolytic effect in hMADS adipocytes. To validate this finding, we verified that incubating a nonspecific $\operatorname{IgG}$ antibody in control wells did not affect basal lipolysis (data not shown). We finally wondered whether this lipolytic effect of GDF15 was a human specificity. We measured gene expression of both GFRAL and GDF15 in whole brain, perigonadic 
A

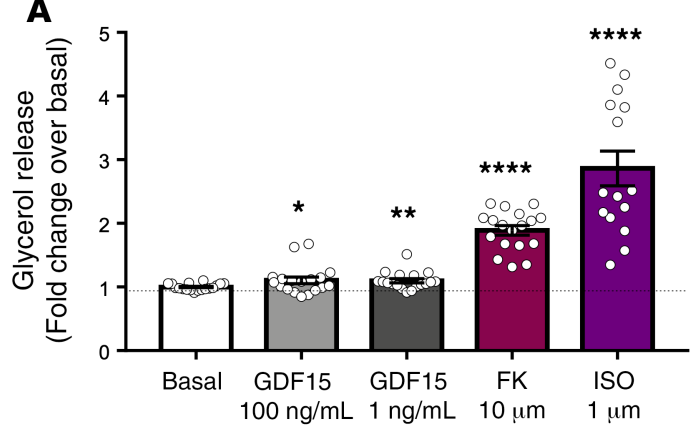

C

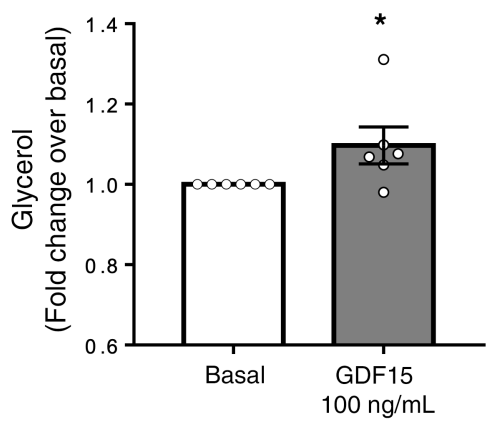

E

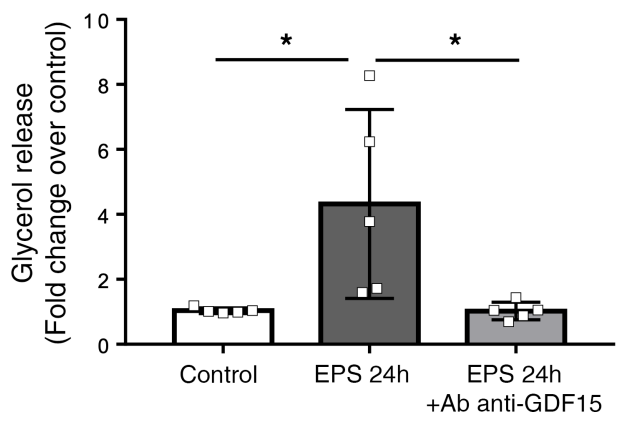

B

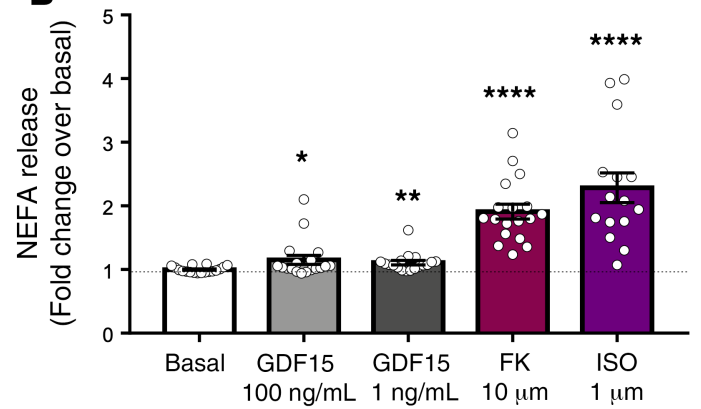

D

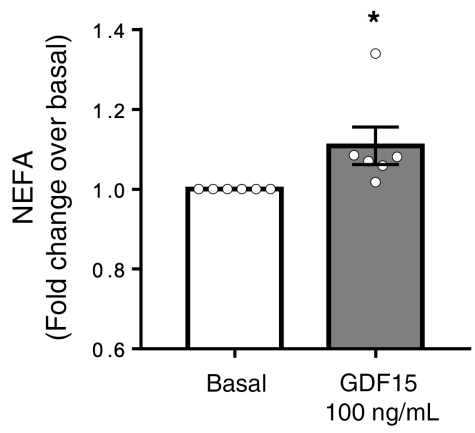

$\mathbf{F}$

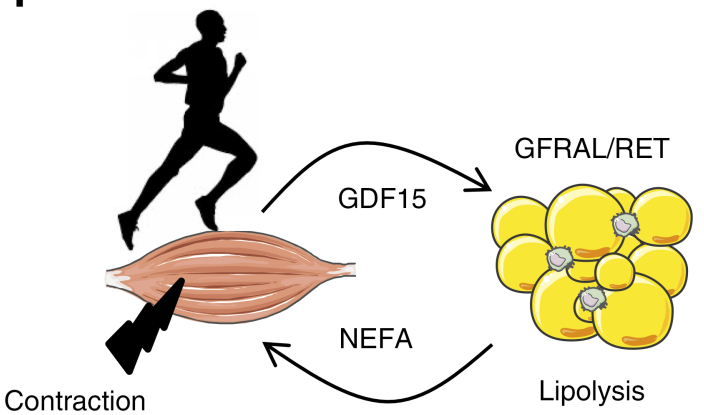

Figure 5. GDF15 promotes lipolysis in human adipose tissue. (A and B) Lipolytic response measured by glycerol release (A) and nonesterified fatty acid release (B) in human abdominal subcutaneous adipose tissue explants in basal condition and in response to 1 and $100 \mathrm{ng} / \mathrm{ml}$ recombinant human GDF15, $10 \mu \mathrm{M}$ forskolin (FK), and $1 \mu \mathrm{M}$ isoprenaline (ISO). Data are expressed as mean $\pm \mathrm{SEM}\left(n=18,3\right.$ replicates of 6 independent donors). ${ }^{*} P<0.05$, ${ }^{* *} P<0.01$ compared by unpaired Student's $t$ test. (C and $\mathbf{D}$ ) Lipolytic response measured by glycerol release (C) and nonesterified fatty acid release (D) in human abdominal subcutaneous adipose tissue explants in basal condition and in response to $100 \mathrm{ng} / \mathrm{ml}$ recombinant human GDF15. Data are expressed as mean \pm SEM ( $n=6$ independent donors). ${ }^{*} P<0.05$ compared by unpaired Student's $t$ test. (E) Conditioned media of EPS24h-stimulated myotubes were applied on differentiated hMADS adipocytes for 24 hours to measure glycerol output, a surrogate of lipolysis, in absence or presence of a GDF15-neutralizing antibody. Data are expressed as mean \pm SEM $(n=5)$. ${ }^{*} P<0.05$ compared with control by 1 -way ANOVA. (F) Integrative model of the skeletal muscle-adipose tissue crosstalk orchestrated by GDF15. Upon muscle contraction, GDF15 is released, reaching its target receptors GFRAL/ RET on adipose tissue to activate lipolysis, which releases nonesterified fatty acid, which further serves as fuel to supply muscle contraction.

white adipose tissue, and skeletal muscle of adult female mice. Although GDF15 was expressed in all 3 organs (Supplemental Figure 5A), GFRAL was only detectable in brain (Supplemental Figure 5B), thus confirming recent findings (16).

Altogether, our data provide the first evidence to our knowledge that GDF15 can target human adipose tissue to trigger lipolysis.

\section{Discussion}

Investigators have been searching for a link between muscle contraction and metabolic changes in other organs, such as the liver and the adipose tissue, for a few decades. The targets of an "exercise factor" have been humoral proteins released from skeletal muscle during contraction that mediate the adaptations of chronic exercise training $(4,20)$. This has suggested a novel paradigm in which skeletal muscle is an endocrine organ 
producing and releasing hormones, i.e., myokines, which exert endocrine effects on remote organs $(4,21)$. We here provide compelling evidence that GDF15 behaves as a exerkine involved in a crosstalk between skeletal muscle and adipose tissue (Figure $5 \mathrm{~F}$ ). We show that the cellular stress mediated by skeletal muscle contraction can be translated into a systemic response through GDF15-mediated lipolysis in white adipocytes.

As we hypothesized that skeletal muscle contraction can release a cellular stress signal able to induce lipolysis, we developed an experimental model of exercise in cultured human myotubes. Human primary culture of skeletal muscle cells has been widely used by several investigators (22-26) and provides a powerful tool for mechanistic studies of skeletal muscle and study muscle autonomous adaptations in the absence of the systemic milieu (27). EPS of human myotubes can be used to induce forced contraction and mimic exercise in vitro (28). Although some studies have used EPS to assess individual variability in biological response to exercise in vitro $(29,30)$ and to identify a potentially novel secreted factors by skeletal muscle contraction (31-33), very few have investigated how closely EPS recapitulates the metabolic consequences of exercise. Here, we developed two exercise models: one acute high-intensity exercise model that recapitulates the expected physiological responses with strong glycogen depletion and high lactate production (34) and one chronic moderate exercise model that more closely mimics the biological adaptations of endurance exercise training with increased basal glycogen storage along with an upregulation of substrate oxidation rates (35).

To identify potential muscle contraction-derived lipolytic factors, we analyzed CM from our two exercise models with human adipocytes. Remarkably, CM from both exercise models increased basal lipolysis. This effect was quite modest for the acute intense exercise model but more pronounced for the chronic moderate exercise model. Thus, a muscle contraction-derived factor contained in CM has the potential to promote lipolysis. Among the currently known myokines, only IL-6 has been previously suggested to induce lipolysis in humans (36). After this, the same authors proposed that muscle-derived IL-6 does not promote fat mobilization from subcutaneous adipose tissue, at least at moderate exercise intensity (37), but rather selectively stimulates fat metabolism in human skeletal muscle (38). This observation was corroborated by in vitro work in adipose tissue explants showing that IL-6 inhibits lipoprotein lipase activity (39). Altogether, most evidence so far indicates that IL- 6 does not acutely stimulate lipolysis in human adipose tissue. In order to unravel novel putative lipolytic myokines, we performed a proteomic screen of EPS CM and identified GDF15 as a significantly upregulated protein in our two in vitro exercise models. GDF15 mRNA levels rise within 30 minutes in response to muscle contraction, followed by an elevated secretion by 1 hour. GDF15 gene expression was also induced by pharmacological exercise mimetics, such as ionomycin and the PPAR $\beta$ agonist GW0742, but remained insensitive to activation of cAMP-signaling by forskolin. This indicates that other molecular pathways related to exercise are involved in GDF15 regulation beyond muscle contraction. As GDF15 is a stress-responsive factor, components of the integrated stress response pathway, including the protein kinase $\mathrm{R}$-like (PKR-like) endoplasmic reticulum kinase (PERK), the eukaryotic translation initiation factor $2-\alpha(\mathrm{EIF} 2 \alpha)$, the activating transcription factor 4 (ATF4), and the CCAAT-enhancer-binding protein homologous protein (CHOP) may be involved (11). A few studies have already suggested that this integrated stress response pathway is activated by exercise $(40,41)$. Thus, several molecular pathways may be involved in the regulation of GDF15 expression/secretion in skeletal muscle.

We consolidated our in vitro findings showing that GDF15 mRNA levels rise from 2- to 20-fold in skeletal muscle of lean healthy subjects in response to acute aerobic exercise. This local rise of GDF15 in skeletal muscle was paralleled by an increase of circulating GDF15 levels during endurance and sprint interval exercise in lean healthy volunteers. Although circulating GDF15 can originate from other tissue sources, it is tempting to speculate, based on our data, that contracting skeletal muscle contributes to increase circulating GDF15 levels during exercise. This is in contrast with a recent study suggesting that the rise of circulating GDF15 during exhaustive exercise appears independent of skeletal muscle (19). Alternatively, muscle-derived GDF15 could play a paracrine role within skeletal muscle to target intramuscular adipose tissue. Obesity did not alter exercise-induced circulating GDF15, but, interestingly, lifestyle intervention-induced weight loss in middle-aged and elderly obese individuals significantly increased baseline circulating GDF15 levels. The rise of plasma GDF15 levels was positively related to weight loss in response to the lifestyle program in elderly obese people, again highlighting a link between GDF15 and adipose tissue loss. Emmerson et al. observed that human subcutaneous adipose tissue is a significant site of expression of the GDF15 receptor GFRAL (16). We therefore went on to hypothesize that GDF15 could target adipose tissue to promote lipolysis.

GDF15 is a cellular stress-responsive hormone that has recently gained much interest as antiobesity therapy $(42,43)$. Recent studies indicate that GDF15 binds to a heterodimeric receptor complex composed 
of GFRAL and RET, with expression restricted to the brainstem in mice (14-17). We here provide evidence that GFRAL and RET are substantially expressed in human adipocytes. A comparable expression is also found in the stroma vascular fraction of adipose tissue, and further cell-type specific analyses revealed that both GFRAL and RET are predominantly expressed in preadipocytes compared with macrophages and lymphocytes. In contrast, GFRAL expression is skeletal muscle tissue is virtually null, thus excluding a potential autocrine role of GDF15. Our data are consistent with the Genotype-Tissue Expression public database, indicating that GFRAL is predominantly expressed in human subcutaneous adipose tissue. Of interest, GFRAL and RET gene expression in subcutaneous abdominal adipose tissue is not influenced by adiposity and obesity grade. Adipose GFRAL and RET gene expression correlated positively with plasma triglycerides, HOMA-IR, and the Matsuda index. This is consistent with the recent observation that GDF15 behaves as a nutritional stress signal induced by long-term high-fat feeding and metabolic disturbances in mice (9-11). In line with an effect of GDF15 on adipose tissue, adipose RET gene expression was inversely related to BMI. Consistently, we here show that a physiological concentration of rhGDF15 induces lipolysis in a comparable range as forskolin in human subcutaneous abdominal adipose tissue explants. In our clinical studies and other studies, circulating GDF15 typically ranged between 0.3 and $1 \mathrm{ng} / \mathrm{ml}(43,44)$. Higher supraphysiological concentrations of up to $100 \mathrm{ng} / \mathrm{ml}$, as observed in cancer cachexia (44), did not further increase lipolysis. Most importantly, neutralization of GDF15 in EPS24h CM with a monoclonal antibody completely abrogated lipolysis, implying that GDF15 triggers most if not all the lipolytic response in CM from EPS-stimulated human myotubes. The higher lipolytic response observed in EPS24h CM could be explained by the nearly 2-fold greater concentration of GDF15 accumulated in the medium over 24 hours. Adipocyte lipolysis is a major physiological process of fuel supply in conditions of increased energy needs, such as exercise $(45,46)$. Our work identifies GDF15 as a potentially novel crosstalk homeostatic hormone between contracting skeletal muscles and adipose tissue in humans.

In summary, GDF15 is an exercise factor involved in a homeostatic regulation loop between contracting skeletal muscles and adipose tissue. Our data suggest that GDF15 is rapidly produced upon skeletal muscle contraction and exercise mimetics. Unlike in mice, GFRAL and RET expression is not highly specific to the caudal brain and displays a nonnegligible expression in human adipose tissue. As GDF15 has emerged as an interesting antiobesity therapy through its central effects on appetite suppression, potential peripheral effects should not be neglected. Hence, future studies of the effects of GDF15 in humans are highly needed.

\section{Methods}

GDF15 protein level determination. GDF15 protein levels in cultured CM and plasma samples were determined by ELISA (R\&D Systems).

Lipolysis assay. Whole-cell lipolysis in differentiated hMADS adipocytes was assessed in control media and EPS CM supplemented with 2\% BSA. The media were changed, and following 3 hours of treatment, glycerol and NEFAs released in the media were measured using commercially available kits (free glycerol reagent, MilliporeSigma; NEFA-C, Wako Chemicals). Data were normalized to total cell protein content.

Thirty g of subcutaneous abdominal adipose tissue were cut with scissors under aseptic conditions into small pieces weighing approximately $10 \mathrm{mg}$ or less. In all experiments, pieces were washed 3 times with sterile PBS to remove blood cells. The pieces of adipose tissue were centrifuged for 1 minute at $300 \mathrm{~g}$ at room temperature between each wash. Then, $20 \mathrm{~g}$ of the pieces of tissue were resuspended in $50 \mathrm{ml}$ fresh medium containing $10 \%$ FCS. The culture medium used was DMEM F12 (Cambrex) containing $33 \mu \mathrm{mol} / 1$ biotin, $17 \mu \mathrm{mol} / 1$ pantothenate, and $50 \mu \mathrm{g} / \mathrm{ml}$ gentamicin. The pieces $(200 \mathrm{mg} / \mathrm{ml})$ were incubated for 4 hours with rhGDF15 concentrations of 1 and $100 \mathrm{ng} / \mathrm{ml}$ and forskolin $(10 \mu \mathrm{M})$ as positive control. Aliquots of the medium were taken after 4 hours of incubation and stored at $-80^{\circ} \mathrm{C}$ for glycerol and NEFAs measurements as described above. The pieces were washed 3 times with PBS, drained, and weighed for data normalization.

Adipose tissue fractionation. Briefly, after collagenase digestion $(250 \mathrm{U} / \mathrm{mL}$ in PBS, $2 \% \mathrm{BSA}, \mathrm{pH} 7.4$, vol/ vol) of the adipose tissue for 20 minutes at $37^{\circ} \mathrm{C}$, the floating mature adipocytes were separated from the SVF. Then, the different SVF cells were isolated using an immunoselection/depletion approach that used magnetic microbeads coupled to specific CD antibodies (CD31, CD34, CD14), which are membrane cell markers to select the different SVF cell types.

The preadipocytes were $\mathrm{CD} 34^{+}$and $\mathrm{CD} 31^{-}$cells. The $\mathrm{CD} 34^{-}$were immune cells (macrophages that are also $\mathrm{CD} 14^{+}$cells and lymphocytes that are CD14- cells) as described in ref. 47. 
Skeletal muscle cell primary culture. Satellite cells from rectus abdominis of healthy male subjects (age 34.3 \pm 2.5 years, BMI $26.0 \pm 1.4 \mathrm{~kg} / \mathrm{m}^{2}$, fasting glucose $5.0 \pm 0.2 \mathrm{mM}$ ) were provided by Arild C. Rustan (Oslo University, Oslo, Norway). Satellite cells were isolated by trypsin digestion, preplated on an uncoated petri dish for 1 hour to remove fibroblasts, and subsequently transferred to T-25 collagen-coated flasks in DMEM low glucose $(1 \mathrm{~g} / \mathrm{L})$ supplemented with $16 \%$ FBS and various factors (human epidermal growth factor, BSA, dexamethasone, gentamicin, fungizone, fetuin) as previously described (48). Cells from several donors were pooled and grown at $37^{\circ} \mathrm{C}$ in a humidified atmosphere of $5 \% \mathrm{CO}_{2}$. Differentiation of myoblasts (i.e., activated satellite cells) into myotubes was initiated at approximately $80 \%-90 \%$ confluence, by switching to $\alpha$-Minimum Essential Medium with $2 \%$ penicillin-streptomycin, $2 \%$ FBS, and fetuin $(0.5 \mathrm{mg} /$ $\mathrm{ml})$. The media were changed every other day, and cells were grown up to 5 days.

EPS. Skeletal muscle cells were fully differentiated for 4 days, and EPS was then applied using a C-Pace EP multichannel culture pacer (IonOptix) for 3 hours (acute intense exercise model) with a protocol consisting of 24-ms pulses at $10 \mathrm{~V}$, with a frequency of $0.5 \mathrm{~Hz}$, or for 24 hours (chronic moderate exercise model) with a protocol consisting of 2 -ms pulses at $10 \mathrm{~V}$, with a frequency of $0.1 \mathrm{~Hz}$. Respective control media from unstimulated skeletal muscle cells were obtained at 3 hours and 24 hours in paired cultures. After completion of EPS, CM were collected for proteomic screening and CM experiments with hMADS adipocytes. The EPS protocol did not induce any visible cell detachment, and assessment of the cytotoxic effect of EPS based on the release of adenylate kinase in culture media using a colorimetric cytotoxicity assay (ToxiLight Non-Destructive Cytotoxicity BioAssay Kit) showed that adenylate kinase activity was not significantly changed in medium from stimulated compared with unstimulated myotubes.

Culture of hMADS cells. hMADS cells were cultured and maintained in proliferation medium (1 g/1 DMEM low glucose; 10\% FBS; 2 mM L-glutamine; 10 mM HEPES buffer; 50 units/ml penicillin; $50 \mathrm{mg} / \mathrm{ml}$ streptomycin; supplemented with $2.5 \mathrm{ng} / \mathrm{ml}$ human FGF2) as previously described (49). The cells were inoculated in 6-well plates at a density of $44,000 \mathrm{cells} / \mathrm{ml}$ and $\mathrm{kept}$ at $37^{\circ} \mathrm{C}$ in $5 \%$ $\mathrm{CO}_{2}$. Six days after seeding, FGF2 was removed from proliferation medium. On the next day (day 0 ), the cells were incubated in differentiation medium (serum-free proliferation medium/Ham's F-12 medium containing $10 \mu \mathrm{g} / \mathrm{ml}$ transferrin, $10 \mathrm{nM}$ insulin, $0.2 \mathrm{nM}$ triiodothyronine, $100 \mu \mathrm{M}$ 3-isobutyl-1- methylxanthine, $1 \mu \mathrm{M}$ dexamethasone, and $100 \mathrm{nM}$ rosiglitazone). At day 3 , dexamethasone and 3-isobutyl-1-methylxanthine were omitted from differentiation medium, and at day 10, rosiglitazone was also omitted. Lipolysis experiments with EPS CM and the GDF15-neutralizing antibody were carried out at day 14. Human FGF2, insulin, triiodothyronine, transferrin, 3-isobutyl-1-methylxanthine, and dexamethasone were from MilliporeSigma; L-glutamine, penicillin, and streptomycin were from Invitrogen; HEPES, DMEM low glucose, and Ham's F-12 medium were from Lonza; and rosiglitazone from was Alexis Biochemicals.

Determination of glucose metabolism. Total glycogen content was determined spectrophotometrically after complete hydrolysis into glucose by the $\alpha$-amyloglucosidase as previously described (50). For glycogen synthesis experiments, myotubes were preincubated with a glucose- and serum-free medium for 90 minutes and then exposed to DMEM supplemented with $\mathrm{D}\left[\mathrm{U}-{ }^{14} \mathrm{C}\right]$ glucose $(1 \mu \mathrm{Ci} / \mathrm{ml}$; PerkinElmer $)$ for 3 hours. Following incubation, glucose oxidation was determined by counting of ${ }^{14} \mathrm{CO}^{2}$ released into the culture medium. The cells were then solubilized in $\mathrm{KOH} 30 \%$, and glycogen synthesis was determined as previously described (26).

Determination of palmitate oxidation. Myotubes were preincubated for 3 hours with $\left[1-{ }^{14} \mathrm{C}\right]$ palmitate $(1$ $\mu \mathrm{Ci} / \mathrm{mL}$; PerkinElmer) and nonlabeled (cold) palmitate (100 $\mu \mathrm{M}$ final concentration). Palmitate was coupled to a fatty acid-free BSA in a molar ratio of 5:1. After incubation, ${ }^{14} \mathrm{CO}^{2}$ and ${ }^{14} \mathrm{C}$-acid soluble metabolite were measured as previously described (51). All assays were performed in duplicate, and data were normalized to cell protein content.

Mass spectrometry-based quantitative analysis of myotube secretome. Methods are provided in the Supplemental Methods. After protein extraction from culture media ( $n=4$ per group), 1 sample pool, comprising equal amounts of all protein extracts, was generated for quality assessment of LC-MS/MS. Individual samples and the sample pool were electrophoresed using SDS-PAGE gels. Five protein bands ( $2 \mathrm{~mm}$ each) per lane were excised from the gels, and proteins were in-gel reduced and alkylated using an automatic pipetting device (MassPrep, Waters) and then digested at $37^{\circ} \mathrm{C}$ overnight with trypsin (Promega). A set of reference peptides (Indexed Retention Time [iRT] Kit, Biognosys) was added to the resulting peptides to allow the stability of instrument performances to be measured for QC purposes. 
Samples were analyzed on a nano-ultraperformance LC system (nanoAcquity, Waters) coupled to a quadrupole-Orbitrap hybrid mass spectrometer (Q-Exactive plus, Thermo Fisher Scientific). Mass spectrometry data were processed using MaxQuant software (v.1.5.8.3, Max Planck Institute of Biochemistry, Martinsried, Germany) (52). Peak lists were created using default parameters and searched using the Andromeda search engine (revert mode) implemented in MaxQuant against a protein database created using the MSDA software suite (53). The database contained human protein sequences (Swiss-Prot; https://www.uniprot.org/ taxonomy/; taxonomy ID, 9606; 20,195 entries), which were downloaded in October 2017. Sequences of common contaminants, such as keratins and trypsin (247 entries), were added to the database (contaminants.fasta included in MaxQuant). A false discovery rate of $1 \%$ for both peptide spectrum matches (minimum length of 7 amino acids) and proteins was accepted during identification. Regarding quantification, data normalization and protein abundance estimation were performed using the label-free quantification (LFQ; ref. 52) option offered in MaxQuant. Proteins identified with only 1 unique peptide were not considered for quantification. Only proteins with at least 3 of 4 valid values per group as well as the ones absent (i.e., 0 valid values) in samples from a given group were kept for further analysis. The MS proteomics data have been deposited to the ProteomeXchange Consortium via the Pride partner repository with the dataset identifier PXD014126.

QC-related measurements showed that HPLC performance remained good and stable throughout the whole experiment, with a median coefficient of variation of $0.5 \%$ concerning retention times of all iRT peptides when considering all injections. The reproducibility of quantitative data was also satisfactory, because we recorded low median coefficients of variation for MaxQuant-derived LFQ values of all quantified proteins within each of the 4 experimental groups (31\%) and in the sample pool of all samples injected repeatedly during the course of MS-based analyses (21\%).

Real-time $q P C R$. Total RNA from cultured myotubes or vastus lateralis muscle was isolated using Qiagen RNeasy mini kit according to manufacturer's instructions (Qiagen). The quantity of RNA was determined on a Nanodrop ND-1000 (Thermo Scientific). Reverse-transcriptase PCR was performed on a Techne PCR System TC-412 using the Multiscribe Reverse Transcriptase method (Applied Biosystems). Real-time quantitative PCR (qPCR) was performed to determine cDNA content. All primers were bought from Applied Biosystems and were as follows: $18 S$ (Taqman assay ID: Hs99999901_s1), GDF15 (Hs00171132_m1), GFRAL (Hs01087628_m1), RET (Hs01120030_m1), IL6 (Hs00985639_m1), IL15 (Hs01003716_m1), FGF21 (Hs00173927_m1), myostatin (Hs00976237_m1), FNDC5 (Hs00401006_m1), apelin (Hs00175572_m1), and $B D N F$ (Hs03805848_m1). The amplification reaction was performed in duplicate on 20 ng cDNA in 96-well reaction plates on a StepOnePlus system (Applied Biosystems). All expression data were normalized by the $2(\triangle \mathrm{Ct})$ method using $18 S$ or PUM1 as internal controls for cell type or whole adipose tissue data, respectively.

Statistics. All statistical analyses were performed using GraphPad Prism 7.0 for Windows. Normal distribution and homogeneity of variance of the data were tested using Shapiro-Wilk and F tests, respectively. Two-tailed Student's unpaired and paired $t$ tests were performed to determine differences between treatments. Two-way ANOVA and Bonferroni's post hoc tests were used when appropriate. All values in figures and tables are presented as mean \pm SEM. Statistical significance was set at $P \leq 0.05$.

Study approval. All protocols were approved by the appropriate institutional review boards and ethics committees (Toulouse Hospital and Dublin City University), and all subjects gave written informed consent.

Author contributions

CL, AP, EM, DC, BL, PM, JV, NB, CF, MM, DL, IH, CT, EM, SCB, VB, GT, JLG, AB, DL, NV, FB, SB, IDG, DO, and CM researched data and edited the manuscript. CL, DO, FB, and CM contributed to study design and revised the manuscript. $\mathrm{CL}$ and $\mathrm{CM}$ wrote the manuscript.

\section{Acknowledgments}

Authors are very grateful to the study participants and to the plastic surgery department of the Toulouse Hospital for providing adipose tissue samples. We particularly thank Corinne Lefort and JeanMarc Strub for technical assistance and François Crampes for insightful discussions and critical reading of the manuscript. CL is currently supported by a postdoctoral fellowship from the French National Space Agency. DL is a member of Institut Universitaire de France. This work was supported by grants from the French Nutrition Society, Region Occitanie/FEDER funds DIABKINE (to CM), the Commission of the European Communities (FP6-513946 DiOGenes), Inserm, Occitanie Region (APRTCN 2013 project MONA), CNRS and Strasbourg University (H2E project), and French Proteomic Infrastructure (ProFI, ANR-10-INSB-08-03). 
Address correspondence to: Cedric Moro, Institute of Metabolic and Cardiovascular Diseases, Inserm UMR1048, CHU Rangueil, BP84225, 1 avenue Jean Poulhès, 31432 Toulouse cedex 4, France. Phone: 33.561.32.5626; Email: cedric.moro@inserm.fr.

1. Hawley JA, Hargreaves M, Joyner MJ, Zierath JR. Integrative biology of exercise. Cell. 2014;159(4):738-749.

2. Pedersen BK, Saltin B. Exercise as medicine - evidence for prescribing exercise as therapy in 26 different chronic diseases. Scand J Med Sci Sports. 2015;25 Suppl 3:1-72.

3. Karstoft K, Pedersen BK. Skeletal muscle as a gene regulatory endocrine organ. Curr Opin Clin Nutr Metab Care. 2016;19(4):270-275.

4. Pedersen BK, Febbraio MA. Muscles, exercise and obesity: skeletal muscle as a secretory organ. Nat Rev Endocrinol. 2012;8(8):457-465.

5. Garneau L, Aguer C. Role of myokines in the development of skeletal muscle insulin resistance and related metabolic defects in type 2 diabetes. Diabetes Metab. 2019;45(6):505-516.

6. Görgens SW, Eckardt K, Jensen J, Drevon CA, Eckel J. Exercise and regulation of adipokine and myokine production. Prog Mol Biol Transl Sci. 2015;135:313-336.

7. Catoire M, Kersten S. The search for exercise factors in humans. FASEB J. 2015;29(5):1615-1628

8. Moro C, et al. Influence of lipolysis and fatty acid availability on fuel selection during exercise. J Physiol Biochem. 2014;70(2):583-591.

9. Böttner M, Suter-Crazzolara C, Schober A, Unsicker K. Expression of a novel member of the TGF-beta superfamily, growth/differentiation factor-15/macrophage-inhibiting cytokine-1 (GDF-15/MIC-1) in adult rat tissues. Cell Tissue Res. 1999;297(1):103-110.

10. Fairlie WD, Moore AG, Bauskin AR, Russell PK, Zhang HP, Breit SN. MIC-1 is a novel TGF-beta superfamily cytokine associated with macrophage activation. J Leukoc Biol. 1999;65(1):2-5.

11. Patel S, et al. GDF15 provides an endocrine signal of nutritional stress in mice and humans. Cell Metab. 2019;29(3):707-718.e8.

12. Fujita Y, Taniguchi Y, Shinkai S, Tanaka M, Ito M. Secreted growth differentiation factor 15 as a potential biomarker for mitochondrial dysfunctions in aging and age-related disorders. Geriatr Gerontol Int. 2016;16 Suppl 1:17-29.

13. Wollert KC, Kempf T, Wallentin L. Growth differentiation factor 15 as a biomarker in cardiovascular disease. Clin Chem. 2017;63(1):140-151

14. Hsu JY, et al. Non-homeostatic body weight regulation through a brainstem-restricted receptor for GDF15. Nature. 2017;550(7675):255-259.

15. Yang L, et al. GFRAL is the receptor for GDF15 and is required for the anti-obesity effects of the ligand. Nat Med. 2017;23(10):1158-1166.

16. Emmerson PJ, et al. The metabolic effects of GDF15 are mediated by the orphan receptor GFRAL. Nat Med. 2017;23(10):1215-1219.

17. Mullican SE, et al. GFRAL is the receptor for GDF15 and the ligand promotes weight loss in mice and nonhuman primates. Nat Med. 2017;23(10):1150-1157.

18. Chrysovergis K, et al. NAG-1/GDF-15 prevents obesity by increasing thermogenesis, lipolysis and oxidative metabolism. Int $J$ Obes (Lond). 2014;38(12):1555-1564.

19. Kleinert M, et al. Exercise increases circulating GDF15 in humans. Mol Metab. 2018;9:187-191.

20. Booth FW. Muscle adaptation to exercise: New Saltin's paradigms. Scand J Med Sci Sports. 2015;25 Suppl 4:49-52.

21. Trayhurn P, Drevon CA, Eckel J. Secreted proteins from adipose tissue and skeletal muscle - adipokines, myokines and adipose/ muscle cross-talk. Arch Physiol Biochem. 2011;117(2):47-56.

22. Lund J, et al. Higher lipid turnover and oxidation in cultured human myotubes from athletic versus sedentary young male subjects. Sci Rep. 2018;8(1):17549.

23. Zou K, et al. Altered tricarboxylic acid cycle flux in primary myotubes from severely obese humans. Int J Obes (Lond). 2019;43(4):895-905.

24. Ciaraldi TP, Ryan AJ, Mudaliar SR, Henry RR. Altered myokine secretion is an intrinsic property of skeletal muscle in type 2 diabetes. PLOS ONE. 2016;11(7):e0158209.

25. Jiang LQ, Duque-Guimaraes DE, Machado UF, Zierath JR, Krook A. Altered response of skeletal muscle to IL-6 in type 2 diabetic patients. Diabetes. 2013;62(2):355-361.

26. Laurens $C$, et al. Perilipin 5 fine-tunes lipid oxidation to metabolic demand and protects against lipotoxicity in skeletal muscle. Sci Rep. 2016;6:38310.

27. Aas V, et al. Are cultured human myotubes far from home? Cell Tissue Res. 2013;354(3):671-682.

28. Nikolić $\mathrm{N}$, et al. Electrical pulse stimulation of cultured human skeletal muscle cells as an in vitro model of exercise. PLoS ONE. 2012;7(3):e33203.

29. Feng YZ, et al. Myotubes from lean and severely obese subjects with and without type 2 diabetes respond differently to an in vitro model of exercise. Am J Physiol, Cell Physiol. 2015;308(7):C548-C556.

30. Park S, et al. Electrical pulse stimulation induces differential responses in insulin action in myotubes from severely obese individuals. J Physiol (Lond). 2019;597(2):449-466.

31. Scheler M, et al. Cytokine response of primary human myotubes in an in vitro exercise model. Am J Physiol, Cell Physiol. 2013;305(8):C877-C886.

32. Whitham M, et al. Contraction-induced interleukin-6 gene transcription in skeletal muscle is regulated by c-Jun terminal kinase/activator protein-1. J Biol Chem. 2012;287(14):10771-10779.

33. Görgens SW, Raschke S, Holven KB, Jensen J, Eckardt K, Eckel J. Regulation of follistatin-like protein 1 expression and secretion in primary human skeletal muscle cells. Arch Physiol Biochem. 2013;119(2):75-80.

34. Vandenberghe K, Hespel P, Vanden Eynde B, Lysens R, Richter EA. No effect of glycogen level on glycogen metabolism during high intensity exercise. Med Sci Sports Exerc. 1995;27(9):1278-1283. 
35. Perseghin G, et al. Increased glucose transport-phosphorylation and muscle glycogen synthesis after exercise training in insulin-resistant subjects. N Engl J Med. 1996;335(18):1357-1362.

36. van Hall G, et al. Interleukin-6 stimulates lipolysis and fat oxidation in humans. J Clin Endocrinol Metab. 2003;88(7):3005-3010.

37. Hiscock N, Fischer CP, Sacchetti M, van Hall G, Febbraio MA, Pedersen BK. Recombinant human interleukin-6 infusion during low-intensity exercise does not enhance whole body lipolysis or fat oxidation in humans. Am J Physiol Endocrinol Metab. 2005;289(1):E2-E7.

38. Wolsk E, Mygind H, Grøndahl TS, Pedersen BK, van Hall G. IL-6 selectively stimulates fat metabolism in human skeletal muscle. Am J Physiol Endocrinol Metab. 2010;299(5):E832-E840.

39. Trujillo ME, Sullivan S, Harten I, Schneider SH, Greenberg AS, Fried SK. Interleukin-6 regulates human adipose tissue lipid metabolism and leptin production in vitro. J Clin Endocrinol Metab. 2004;89(11):5577-5582.

40. Wu J, et al. The unfolded protein response mediates adaptation to exercise in skeletal muscle through a PGC-1 $\alpha /$ ATF6 $\alpha$ complex. Cell Metab. 2011;13(2):160-169.

41. Kim HJ, et al. Endoplasmic reticulum stress markers and ubiquitin-proteasome pathway activity in response to a 200-km run. Med Sci Sports Exerc. 2011;43(1):18-25.

42. Chung HK, et al. Growth differentiation factor 15 is a myomitokine governing systemic energy homeostasis. J Cell Biol. 2017;216(1):149-165.

43. Tsai VWW, Husaini Y, Sainsbury A, Brown DA, Breit SN. The MIC-1/GDF15-GFRAL pathway in energy homeostasis: implications for obesity, cachexia, and other associated diseases. Cell Metab. 2018;28(3):353-368.

44. Wolfe LS, Pappius HM, Pokrupa R, Hakim A. Involvement of arachidonic acid metabolites in experimental brain injury. Identi fication of lipoxygenase products in brain. Clinical studies on prostacyclin infusion in acute cerebral ischemia. Adv Prostaglandin Thromboxane Leukot Res. 1985;15:585-588.

45. Arner P, Langin D. Lipolysis in lipid turnover, cancer cachexia, and obesity-induced insulin resistance. Trends Endocrinol Metab. 2014;25(5):255-262.

46. Lafontan M. Historical perspectives in fat cell biology: the fat cell as a model for the investigation of hormonal and metabolic pathways. Am J Physiol, Cell Physiol. 2012;302(2):C327-C359.

47. Maumus M, et al. Native human adipose stromal cells: localization, morphology and phenotype. Int J Obes (Lond). 2011;35(9):1141-1153.

48. Badin PM, et al. Regulation of skeletal muscle lipolysis and oxidative metabolism by the co-lipase CGI-58. J Lipid Res. 2012;53(5):839-848

49. Girousse A, et al. Partial inhibition of adipose tissue lipolysis improves glucose metabolism and insulin sensitivity without alteration of fat mass. PLoS Biol. 2013;11(2):e1001485.

50. Bourlier V, et al. Enhanced glucose metabolism is preserved in cultured primary myotubes from obese donors in response to exercise training. J Clin Endocrinol Metab. 2013;98(9):3739-3747.

51. Engeli S, et al. Natriuretic peptides enhance the oxidative capacity of human skeletal muscle. J Clin Invest. 2012;122(12):4675-4679.

52. Cox J, Hein MY, Luber CA, Paron I, Nagaraj N, Mann M. Accurate proteome-wide label-free quantification by delayed normalization and maximal peptide ratio extraction, termed MaxLFQ. Mol Cell Proteomics. 2014;13(9):2513-2526.

53. Carapito C, et al. MSDA, a proteomics software suite for in-depth mass spectrometry data analysis using grid computing. Proteomics. 2014;14(9):1014-1019. 